\title{
Platysma Sign-A Clinical Manifestation of Infiltration of Platysma and the Subcutaneous Tissue by Large Neck Node
}

\author{
Chaturvedi Pankaj, Agarwal Jai Prakash \\ Department of Head and Neck Oncology, Tata Memorial Hospital, Parel, Mumbai, Maharashtra, India
}

Correspondence: Chaturvedi Pankaj, Associate Professor, Department of Head and Neck Service, Tata Memorial Hospital Dr E Borges Road, Parel, Mumbai-400012, Maharashtra, India, Phone: +912224177189, Fax: +912224146937 e-mail: pankaj37@yahoo.com

\begin{abstract}
We propose a simple clinical sign that is seen in certain patients with bulky nodal metastases. The fundamental basis of this sign is the direct infiltration of platysma by the nodal mass causing contracture of the muscle fibers of platysma called "Platysma sign". The exact definition of this sign is "puckering of the platysma and the overlying skin like an inverted Japanese fan". This puckering always happens below the level of nodes and finishes at the level of the clavicle. This clinical sign can serve as an instant reminder to the clinician and avoid oversight of the skin/platysma infiltration. Assessment of the integrity of the neck skin overlying a large neck nodes must be undertaken while examining a head and neck cancer patient with bulky neck metastasis. Infiltration of platysma or the skin is an important finding in the staging and treatment of the neck disease.
\end{abstract}

Keywords: Platysma, neck node, extracapsular spread, clinical sign, metastasis.

\section{DESCRIPTION}

The platysma is a superficial muscle that shields the sternocleidomastoid muscle, facial artery (near the lower border of the mandible), external jugular vein and several cutaneous nerves. It is a broad muscular sheet originating from the fascia covering the upper parts of the deltoid and pectoralis major muscle. The muscle fibers cross the clavicle and lie obliquely upward and medially along the side of the neck. The anterosuperior fibers interlace with the fibers of the muscle of the opposite side below the symphysis menti, the posterosuperior fibers cross the mandible to get inserted below the oblique line. Some posterior fibers get inserted into the adjoining subcutaneous tissue. It is supplied by the cervical branch of the facial nerve. It draws the corners of the mouth inferiorly and widens it. It also draws the skin of the neck superiorly when teeth are forcefully clenched. Platysma may be directly invaded by the neighboring large metastatic neck nodes.

We propose a simple clinical sign that is seen in certain patients with bulky nodal metastases. The fundamental basis of this sign is direct infiltration of platysma by the nodal mass causing contracture of the muscle fibers of platysma called "Platysma sign". The exact definition of this sign is "puckering of the platysma and the overlying skin like an inverted Japanese fan” (Fig. 1). This puckering always happens below the level of nodes and finishes at the level of the clavicle (Fig. 1). This clinical sign can serve as an instant reminder to the clinician and avoid oversight of the skin/ platysma infiltration.

Assessment of the integrity of the neck skin overlying a large neck node must be undertaken while examining a head and neck cancer patient. Infiltration of platysma or the skin is an important finding in the staging and treatment of the neck disease. The infiltration of the platysma muscle is a sign of aggressive disease and usually seen in presence of

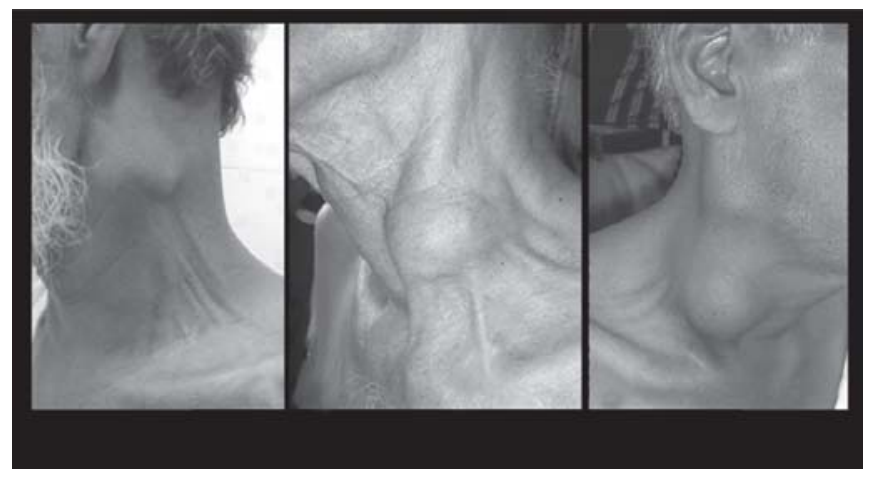

Fig. 1: Platysma sign: Puckering of the skin and underlying platysma in three patients with large metastatic neck nodes. Note that the puckering starts at the level of the node and finishes at the level of the clavicle 
large neck nodes (N2 and N3). Skin or platysma infiltration denotes extracapsular spread that is a proven poor prognostic factor. ${ }^{1,2}$ Involvement of platysma has bearing on the surgical management as well. In case surgery is planned, the involved skin needs to be removed en bloc with the neck dissection specimen. In presence of platysma infiltration, any preservation of the overlying skin is technically and oncologically unsafe. Since the blood vessels run in the subplatysmal plane, a neck skin flap bereft of platysma is prone to ischemic necrosis. Moreover, preserving the overlying skin, when the platysma is involved, may lead to positive soft tissue margin with a higher chances of local failure.
We feel that this is a simple yet improtant clinical sign which will help the head-neck surgeon in their routine clinical practice.

\section{REFERENCES}

1. Ferlito A, Rinaldo A, Devaney KO, MacLennan K, Myers JN, Petruzzelli GJ, Shaha AR, Genden EM, Johnson JT, de Carvalho MB, Myers EN. Prognostic significance of microscopic and macroscopic extracapsular spread from metastatic tumor in the cervical lymph nodes. Oral Oncol Dec 2002;38(8):747-51.

2. Puri SK, Fan CY, Hanna E. Significance of extracapsular lymph node metastases in patients with head and neck squamous cell carcinoma. Curr Opin Otolaryngol Head-Neck Surg Apr 2003;11(2):119-23. 